\title{
L'image-événement
}

\section{Retour sur les lieux de l'evénement : l'image « en creux »}

Return to the Scene of the Event. The Empty Image

\section{Raphaële Bertho}

\section{(2) OpenEdition \\ 12 Journals}

Édition électronique

URL : http://journals.openedition.org/imagesrevues/336

DOI : 10.4000/imagesrevues.336

ISSN : 1778-3801

\section{Éditeur :}

Centre d'Histoire et Théorie des Arts, Groupe d'Anthropologie Historique de l'Occident Médiéval, Laboratoire d'Anthropologie Sociale, UMR 8210 Anthropologie et Histoire des Mondes Antiques

\section{Référence électronique}

Raphaële Bertho, «Retour sur les lieux de l'evénement : l'image « en creux » », Images Re-vues [En ligne], 5 | 2008, mis en ligne le 01 septembre 2008, consulté le 30 janvier 2021. URL : http:// journals.openedition.org/imagesrevues/336 ; DOI : https://doi.org/10.4000/imagesrevues.336

Ce document a été généré automatiquement le 30 janvier 2021.

Images Re-vues est mise à disposition selon les termes de la Licence Creative Commons Attribution Pas d'Utilisation Commerciale 4.0 International. 


\title{
Retour sur les lieux de l'evénement: l'image « en creux »
}

\author{
Return to the Scene of the Event. The Empty Image
}

Raphaële Bertho

1 «Pour qu'il y ait événement, il faut qu'il soit connu $»^{1}$. Mais aussi qu'il soit vu. Si c'est effectivement aux mass médias que l'événement doit de nos jours son existence, c'est désormais par l'image qu'il prend corps. L'événement ne semble pas pouvoir exister s'il n'en existe pas d'images. Nos concitoyens sont comme l'apôtre, ils doivent le voir pour le croire.

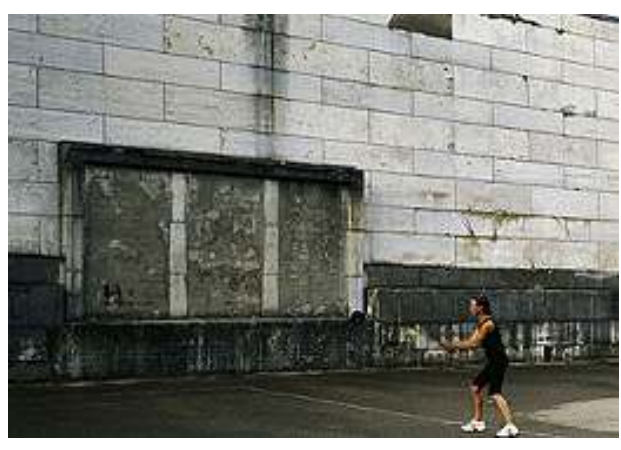
Cette hégémonie du visible transforme les images du photojournalisme en «icônes ». Largement diffusées dans le temps et dans l'espace sur différents supports, elles sont sorties de leur contexte et dépouillées de leur signification historique pour devenir des représentations emblématiques. Au-delà de leur fonction informative ou factuelle, elles deviennent des « images monuments $»^{2}$ et intègrent la mémoire collective ${ }^{3}$.

Une « actualité-mémoire » qui conduit à une forme d'asphyxie du regard, du fait de la production en flux continu des mass médias d'images sur-significatives. Des images de remplissage, de surface, à consommer sur le mode du «zapping mental $»^{4}$. Face à ce débordement, à ce " trop plein », on observe l'apparition d'une posture nouvelle dans le champ de la photographie contemporaine. Comme "en creux» du phénomène médiatique, elles travaillent à l'évocation des grands évènements contemporains tout en étant vides de leur représentation explicite. Des images qui tentent d'interroger la mémoire collective iconologique construite par les médias. L'événement n'investit pas l'image elle-même, mais reste dans une présence latente. Elles ne sont donc pas "creuses», au sens d'absence de sens, mais leur signification est perceptible en " creux », en filigrane. Les auteurs invitent le spectateur à l'investir de sens, à venir 
compléter la représentation par l'interprétation, à percevoir « une image par-devers l'image $»^{5}$.

3 Ces images proposent un retour sur l'événement, au sens propre comme au figuré. Un retour sur les lieux, pour tenter d'ouvrir un espace de réflexion sur, par, et dans l'image. On observe ici le passage d'une stratégie de représentation de l'événement à une stratégie d'évocation du passé. Face à ces images "en creux", il ne s'agit pas seulement d'imaginer, de se figurer un «irréel», selon les mots de Sartre ${ }^{6}$, mais de se représenter un «réel antérieur ». Acte de remémoration qui peut sembler impossible, si l'on n'a jamais connu ce réel antérieur. Mais les images qui circulent autour de nous, issues des mass médias, journaux ou télévision, interfèrent nécessairement avec nos images mentales. "La mémoire n'est pas un écran vierge susceptible de donner naissance à des images purement mentales ${ }^{7}$, comme le remarque Arno Gisinger. Il s'agit, afin de se figurer l'événement, de fouiller dans notre mémoire collective iconologique. Les images "en creux » sont donc intrinsèquement liées aux images «monuments».

Fig.1

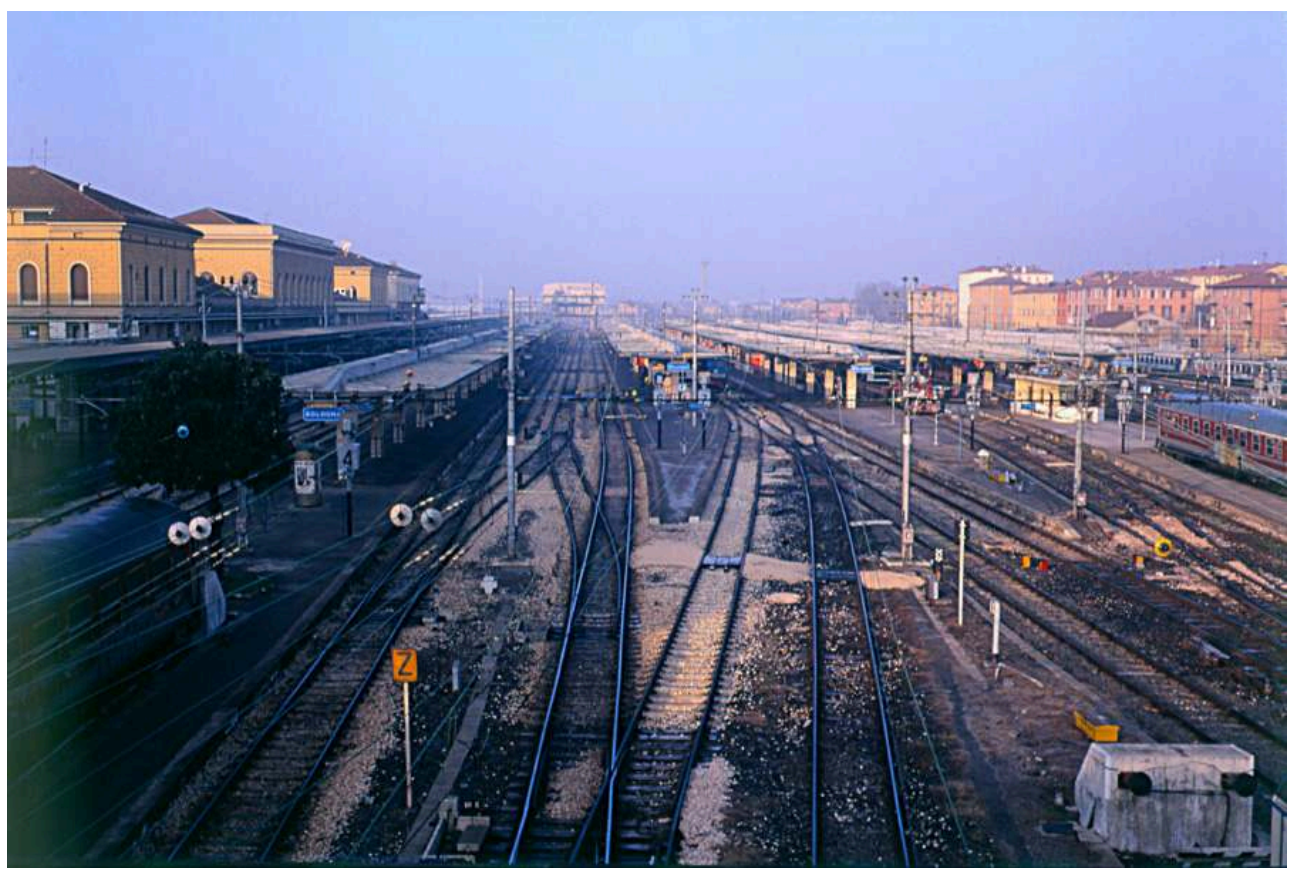

Christophe DRAEGER, Bologne, Italie, 27 décembre 1999, de la série Voyages apocalyptiques (depuis 1994). 20x24cm, C-print framed, 31

(c) Christophe DRAEGER

4 Si les images «en creux » font fréquemment référence aux drames humains qui ont marqué le siècle, cela tient au fait que les guerres et les catastrophes catalysent une double problématique. Ces événements favorisent la «mise en spectacle» par les médias de masse, tout en étant au cœur de la problématique du devoir de mémoire. Ainsi lorsque Christophe Draeger dans son Voyage Apocalyptique revient sur les lieux de la catastrophe, c'est pour en détourner le potentiel spectaculaire. D'une autre manière, Sophie Ristelhueber dans Fait travaille à attester de la réalité d'un conflit que la médiatisation a rendu presque abstrait, allant presque jusqu'à l'invisibilité. Un voyage sur les traces du passé qui conduit Stéphane Duroy et Arno Gisinger à parcourir 
l'Europe, vieux continent marqué par les deux Guerres Mondiales. En dehors du spectaculaire, c'est dans les lieux du quotidien que les deux photographes ancrent leur appel à la mémoire. D'autres lieux sont porteurs d'un passé encore plus chargé, comme Hiroshima et Nagasaki, Auschwitz, Tchernobyl. Ce sont ces lieux « trauma » que Hiromi Tsuchida, Marie-Jeanne Musiol et Guillaume Herbault reviennent arpenter. Les photographes réactivent l'expérience vécue, dans une volonté d'aller au-delà d'un devoir de mémoire paralysant, afin d'intégrer de nouveau ces évènements à notre mémoire collective.

Fig. 2

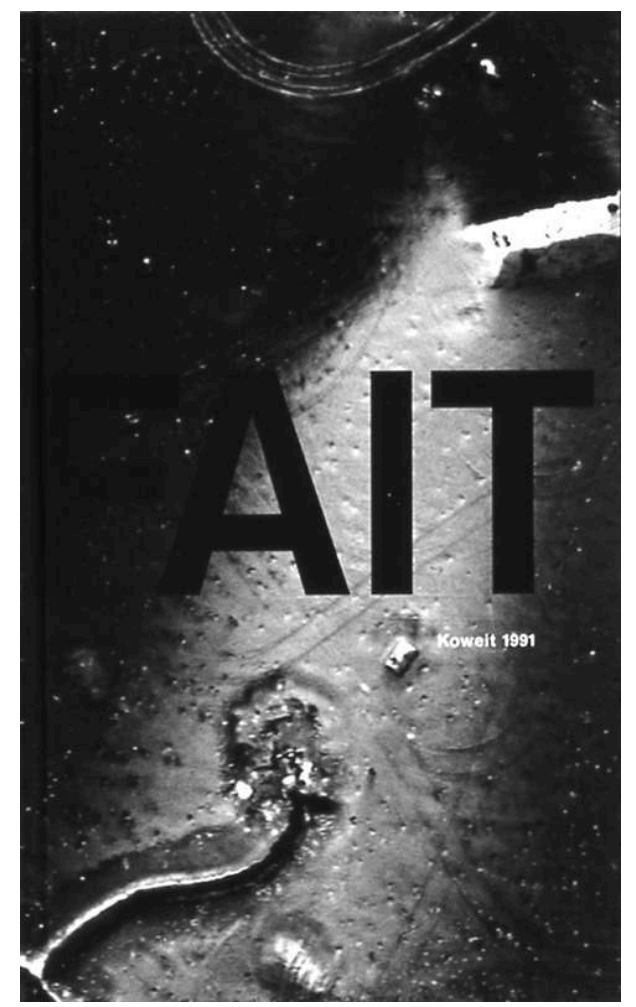

Sophie RISTELHUERBER, Couverture de Fait, Koweit 1991, Paris, Hazan, 1992.

(C) Sophie RISTELHUERBER 


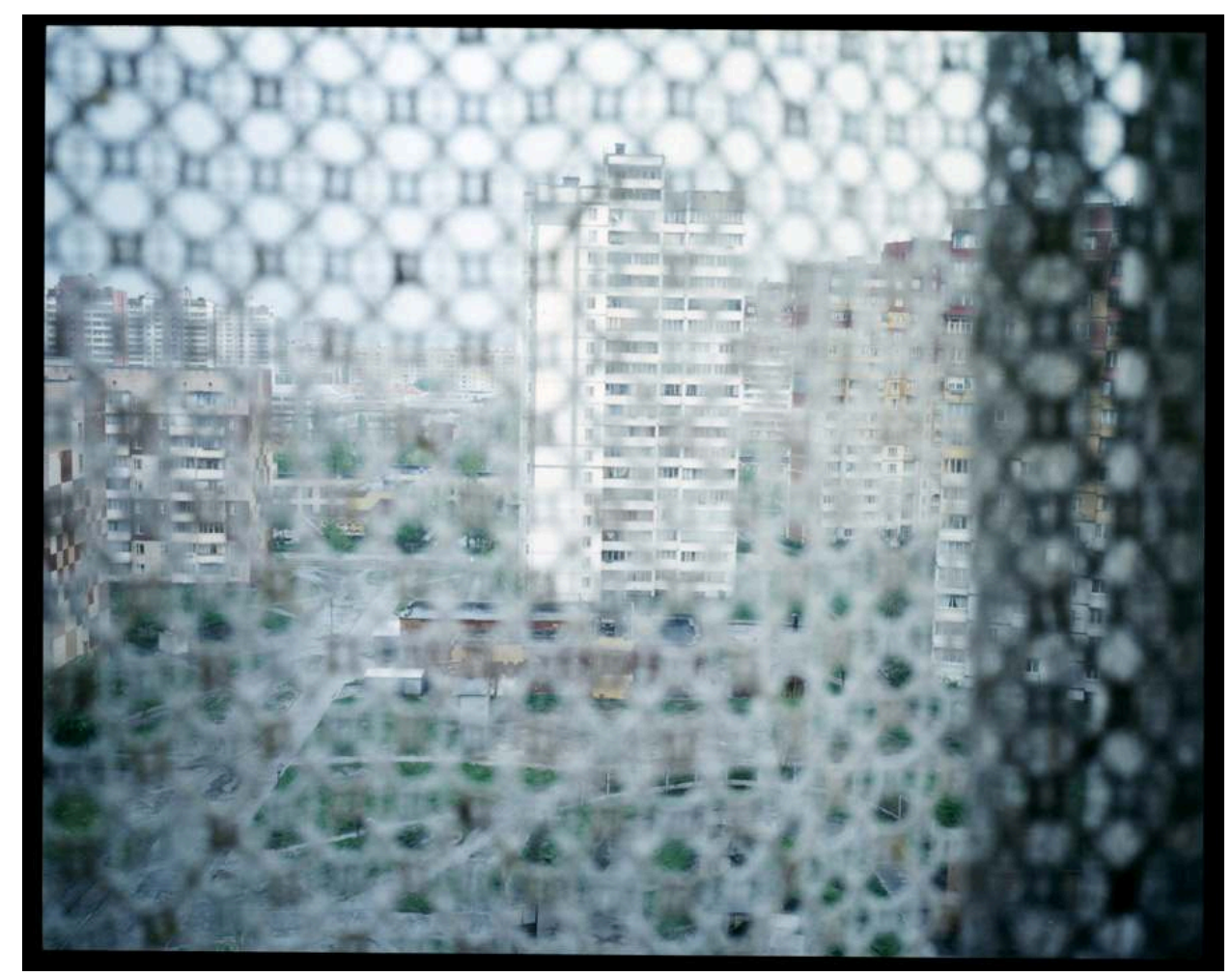

Guillaume HERBAUT, Kiev, quartier limitrophe de Troschina, habité pour les trois-quart par les Tchernobylstys, les anciens habitants de la ville de Tchernobyl, issu de Tchernobylsty, 2003.

(c) Guillaume Herbaut / L'œil Public.

5 Le résultat photographique est « distancié, quasiment déconstruit, afin que chacun puisse imaginer, en fonction de son expérience, ce que l'on ne voit pas $»^{8}$. Une distance qui n'est pas sans rappeler la stratégie du retrait évoquée par Dominique Baqué ${ }^{9}$ a propos des travaux de Jacqueline Salmon ou de Raymond Depardon. Les images « en creux » n'échappent pas totalement à cette notion, sans pour autant y adhérer dans leur ensemble. La stratégie du retrait analysée par Dominique Baqué répond tout autant à une volonté d'" échanger du visible contre de la pensée " qu'à celle de se démarquer $\mathrm{du}$ " voyeurisme » propre à l'image de presse ${ }^{10}$. S'agissant des images «en creux ", elles tendent effectivement à interpeller le spectateur, à susciter une réflexion, une remémoration. Mais il n'est pas question de pudeur comme chez Jacqueline Salmon, ou de promouvoir une photographie des temps faibles à l'instar de Raymond Depardon. Les photographes qui réalisent ces images « en creux » ne présentent aucune velléité de défocaliser la vision de l'événement. Au contraire, ils reviennent sur les lieux, ils réorientent le regard vers ces lieux, théâtres d'événements tragiques. Il ne s'agit pas de critiquer l'affect ou le sensationnalisme liés aux représentations de presse, mais d'opérer un retour au présent. Ils ne se retirent pas, mais font une photographie de l'après. Le «creux » n'est pas une représentation de l'absence ou de la disparition, mais du manque, celui de traces visibles de l'événement. Ce manque est identifié par la mise sous tension de l'image à travers le texte qui lui est associé. Une tension entre le visible, le figuré, « ce qui est là », et l'invisible, ici le passé, « ce qui a été là », voire " ce qui aurait dû être là ». Les images « en creux » s'analysent donc comme un courant de la photographie contemporaine, non pas autonome, mais spécifique. 
Fig. 4

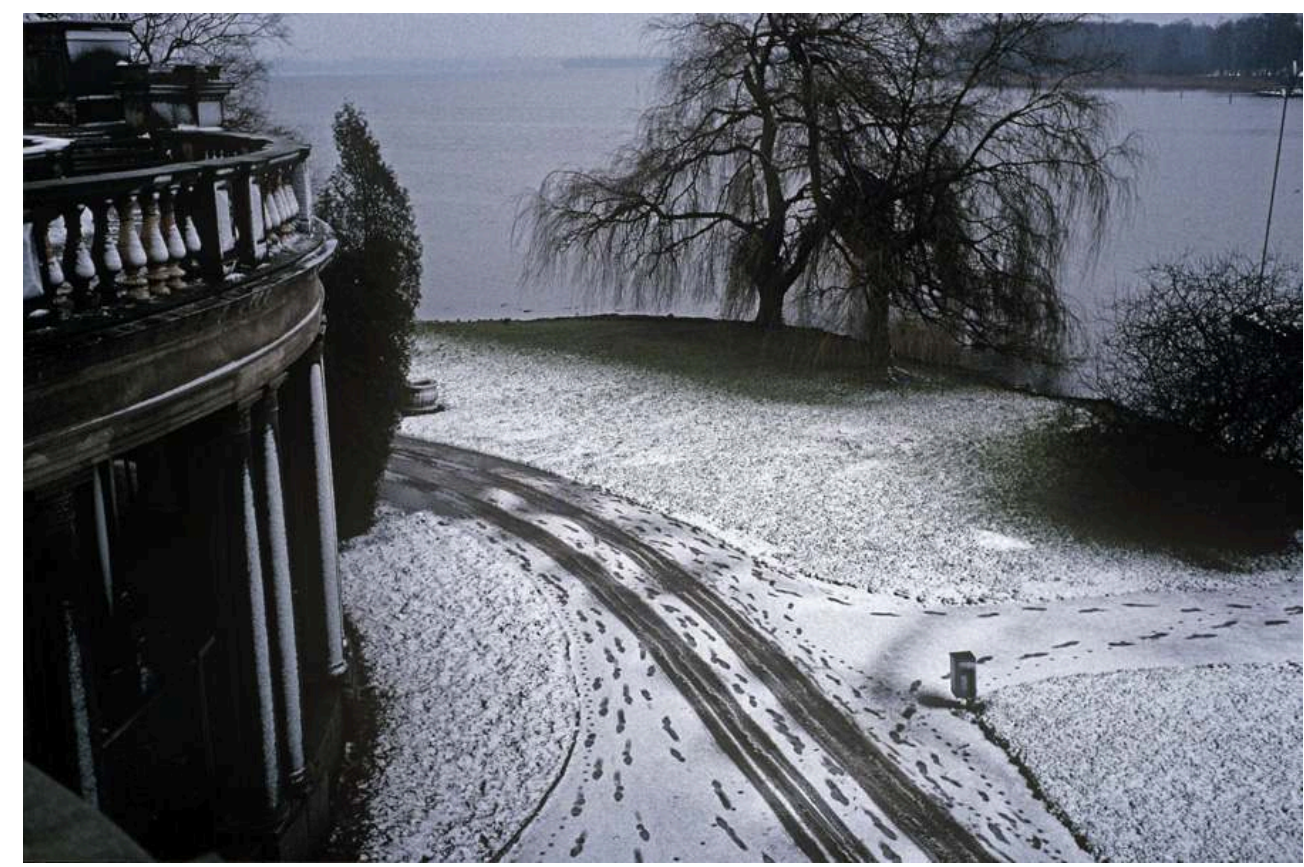

Stéphane DUROY, Schwerin, issue de L'Europe du silence, 1999.

(c) Stéphane Duroy / Agence VU

6 À rebours du poids des mots et du choc des photos, pour reprendre le célèbre slogan d'un magazine à grand tirage, le corpus présenté ici, loin d'être exhaustif, permet néanmoins de cerner les différentes facettes de cette stratégie de l'image " en creux ", dont les éléments fondamentaux peuvent s'analyser comme étant le retour sur les lieux et la mise sous tension de l'image par le texte.

\section{Le retour sur les lieux}

7 Le retour ne s'apparente pas à un pèlerinage, mais plutôt à un arpentage curieux de ce que ces lieux de mémoire recèlent en réalité. Les photographes reviennent sur les lieux comme on va sur le terrain, pour se rendre compte, et se confrontent parfois à une véritable forclusion du paysage.

8 Sophie Ristelhueber, pour réaliser sa série Fait, parcourt durant quatre semaines le désert du Koweït, encore brûlant de la Guerre du Golfe. Cet engagement physique fait, selon elle, partie intégrante de son travail, sans lequel l'œuvre ne peut être pensée. En plus des vols au-dessus du désert, elle tient à marcher au milieu des tranchées, des douilles, des mines, des traces de tanks et des effets personnels abandonnés, dans un investissement physique que Jerôme Sans considère comme "proche de la performance $»^{11}$. De la même manière, l'expérience du lieu est au fondement de l'œuvre de Marie-Jeanne Musiol qui se rend à huit reprises à Auschwitz-Birkenau pour réaliser la série Dans l'ombre de la forêt (Auschwitz-Birkenau). Sa connaissance du site lui permet de s'écarter des lieux balisés, pour aller vers d'autres lieux, sans doute moins éculés. Elle choisit délibérément de photographier, non pas les installations concentrationnaires, mais leur périphérie. 
Quand ces deux photographes décident de taire le déroulement exact de leur expédition, afin que la dimension narrative ne prenne pas le pas sur la puissance évocatrice de l'image, Hiromi Tsuchida adopte le parti pris inverse. Il réalise, entre 1979 et 1983, Hiroshima Monument, en revenant sur le lieu où la bombe atomique a explosé trente-quatre ans plus tôt. L'œuvre se décompose en trois parties, l'une étant consacrée à la recherche des lieux restés intacts lors de l'impact dans la ville de Hiroshima, la seconde dressant le portrait de survivants, et la dernière étant constituée de photographies d'objets rescapés de la bombe. On peut remarquer que cette œuvre, tout comme celle de Marie-Jeanne Musiol, occulte le foyer même de la catastrophe, lequel n'est jamais représenté. On prendra ici le parti de n'étudier que la première partie de Hiroshima Monument, consacrée à l'arpentage de la ville, cette démarche étant la plus pertinente du point de vue de notre étude ${ }^{12}$. Elle se compose d'une série de quarante diptyques constitués d'une photographie et d'un plan de la ville. Sur le plan apparaît une étoile, qui marque le point d'impact de la bombe atomique. Autour de ce centre, des points indiquent les lieux photographiés par Hiromi Tsuschida. Et la carte, identique dans chaque diptyque, s'alimente au fur et à mesure de ces points marquant l'endroit des prises de vues. Ainsi le photographe donne à voir au sein même de l'œuvre ses déplacements dans la ville, quand bien même le déroulement chronologique des déplacements reste fictionnel.

Cette dimension topographique est tout aussi présente dans le travail d'Arno Gisinger consacré à deux camps d'internement ouverts près du Mans pendant la Seconde Guerre Mondiale, Courdecieux et Mulsanne (2006). Il ne reste que peu de traces de ces structures, et ce n'est pas par les mots mais par les plans que le photographe atteste de leur existence. Arno Gisinger imprime des plans d'époque au verso des tirages afin de "démontrer la présence de l'histoire par son absence ${ }^{13}$, faisant de la topographie la « clé » de cet espace de mémoire. 
Fig. 5

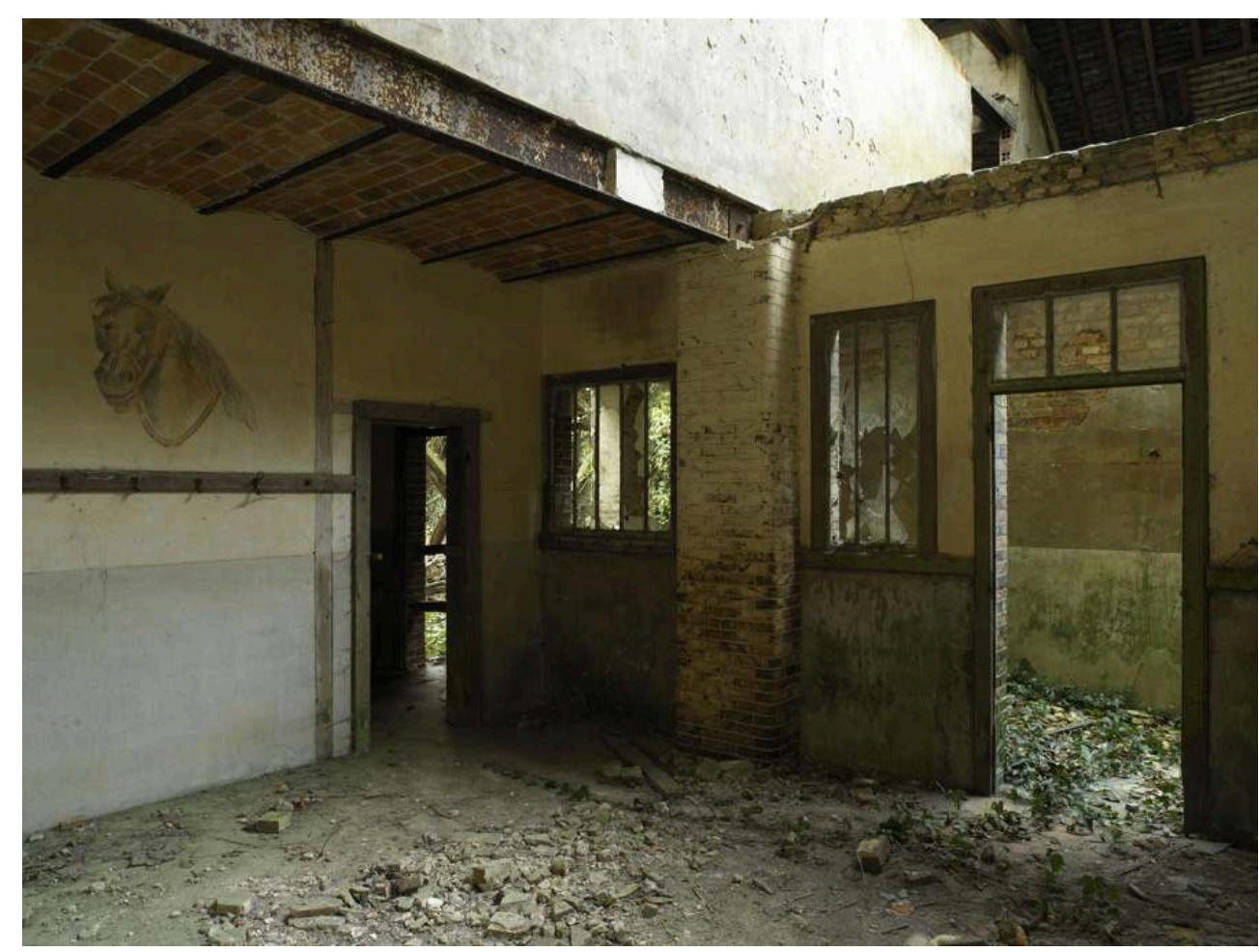

Arno GISINGER, Coudrecieux, 2006.

(C) Arno GISINGER

11 S'il photographie au cœur même d'une ville, Hiromi Tsuchida évite pour autant toute présence humaine sur ses images. Les seuls personnages représentés sont presque invisibles, tapis dans l'ombre de l'image, ou pris dans le flou de leur vitesse. Cette quasiabsence de tout signe de vie, loin d'être une négation de la dimension humaine de l'évènement, semble correspondre à une volonté d'atteindre une certaine intemporalité. Cette représentation des rémanences de l'événement n'est pas sans faire penser à d'anciennes écritures photographiques de l'histoire, quand l'opérateur ne pouvait pas photographier l'événement lui-même du fait d'impératifs techniques. Il représentait alors seulement les preuves de l'événement, ce qui résistait le plus à la perte: les façades noircies par l'incendie, les carcasses laissées à l'abandon, les décombres des bâtiments détruits... Une absence de tout signe de vie qui, éloignant toute possibilité de compassion ou d'identification de la part du spectateur, favorise un retour réflexif sur l'image. En l'absence de toute théâtralité, entendue dans le sens de Fried $^{14}$, qui capte le spectateur, la relation de ce dernier à l'image reste alors ouverte.

Retour sur les lieux, décalage chronologique par rapport à l'événement... Les auteurs de ces images " en creux » pourraient être ici qualifiés d' " archéologues photographes ». Dans ce sens, Arno Gisinger affirme, sans être le seul, mener des recherches en amont de ses prises de vue. L'ensemble des travaux de ce photographe, qui a aussi une formation d'historien, concerne d'une manière plus ou moins lointaine un seul événement: la Seconde Guerre Mondiale. Après s'être intéressé, de manière critique, aux formes de commémoration, le photographe tend aujourd'hui, à travers différentes séries, à rendre visibles les vestiges de l'histoire. Une enquête dans les archives lui permet ainsi de redécouvrir des traces enfouies ou ignorées. Dans ses récents travaux sur la ville de Nuremberg en 2004, Nuremberg, les coulisses du pouvoir et Nuremberg, la 
scène du procès, Arno Gisinger souhaite " exhumer des lieux, des traces qui ne sont pas forcément visibles, ou qui sont totalement oubliées aujourd'hui $»^{15}$. Il ne faut pourtant pas s'y tromper, il ne s'agit pas d'une reconstitution, le photographe ne cherche pas à rendre une atmosphère d'époque dans ses images. Ce retour vers le passé ne s'apparente pas à un voyage dans le temps.

Fig. 6

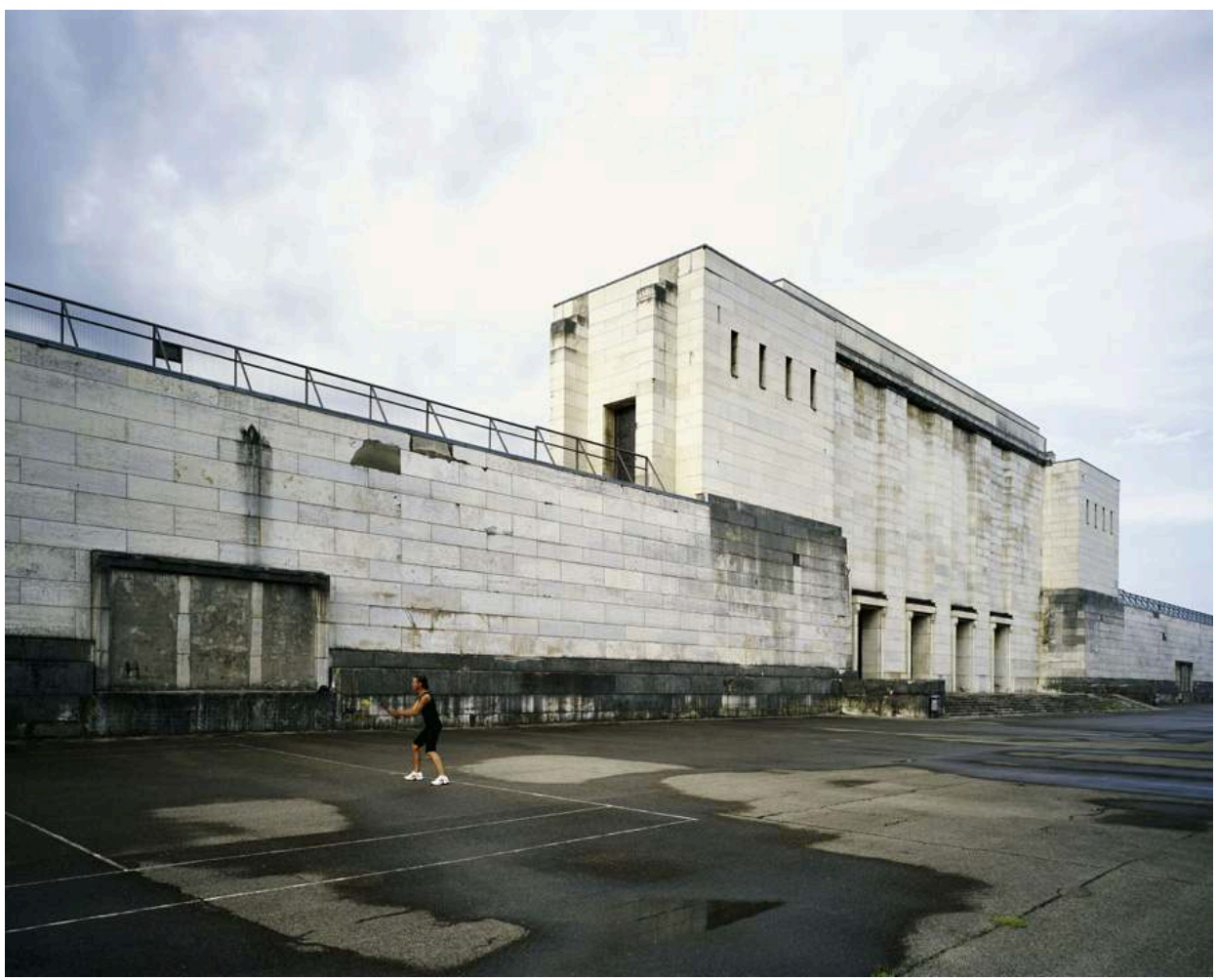

Arno GISINGER, Nuremberg, les coulisses du pouvoir, 2004.

\section{(C) Arno GISINGER}

13 Au contraire, l'image « en creux » s'ancre délibérément dans le présent, et c'est à partir de là qu'elle nous propose de regarder en arrière, mesurant dans le «creux » de la représentation toute la distance qui nous sépare de l'événement. Les photographes se confrontent aux lieux, en évitant toute dramatisation. Le titre choisi par Sophie Ristelhueber, Fait, apparaît comme révélateur.

Il me fallait un titre laconique [...]. Ce que j'ai vu - la guerre - c'est un fait. Les formes que j'ai saisies ont été 'faites' par la guerre, puis par moi. La guerre - et ses formes - ne dit rien d'autre que 'c'est comme ça'"

14 À l'origine des photographies présentées dans Fait, une image aérienne parue dans le Time le 25 février 1991, représente des traces noires d'impact de bombes sur le sol du désert strié de lignes indéfinies. Sophie Ristelhueber décide alors de réaliser des images du désert koweitien, en allant y rechercher les vestiges de cette Guerre du Golfe rendue presque virtuelle par les médias. La photographe alterne vues aériennes et vues au sol et organise une perturbation volontaire de la vision en supprimant pratiquement tout repère d'échelle. Les images se présentent comme des all over, sans horizons, sans limites repérables. Elles ne semblent pas être des photographies de guerre au sens attendu du terme, mais seulement la représentation de longues et profondes blessures 
gravées dans le sable, évoquant, selon un écho indirect, le conflit et ses conséquences. Et pourtant, les vestiges de la bataille sont bien là, au cœur de l'image. Les photographies représentent des tranchées, des traces de bombardement, des carcasses d'engins, et des objets plus personnels, des chaussures ou des couvertures. Ces traces, bien que présentes, sont rendues presque indiscernables par le jeu des échelles, qui empêchent le regard d'adopter un point de vue, et par ce désert au ton ocre qui emplit la photographie et la sature.

L'artillerie comme les divers accessoires de guerre laissés par l'armée lors de son repli sont mués par la caméra en simples objets banals $[. . .]^{17}$.

Ironie du travail, les vestiges sont sous nos yeux, mais d'une telle manière que nous ne savons les voir. Ce glissement perceptif requiert de la part du spectateur une attention accrue, puisqu'il ne peut se contenter de passer rapidement son regard sur les images pour en saisir le sens.

De la même façon, les vestiges du drame nucléaire de Hiroshima sont au cœur des images de Hiromi Tsuchida, sans être perceptibles en tant que tels. Paradoxalement intacts pour la plupart, des cerisiers, des pins ou des camphriers, une banque ou une ancienne gare de triage, des ponts de pierre ou d'acier, autant de sites d'incarnation de la mémoire collective. Ils sont noyés dans la ville contemporaine, indiscernables comme l'étaient ceux du désert koweitien. Marie-Jeanne Musiol, au contraire, en revenant sur les lieux du camp d'extermination et de concentration d'AuschwitzBirkenau, prend le parti de totalement détourner le regard. Dans son œuvre intitulée Dans l'ombre de la forêt (Auschwitz Birkenau), elle se situe à la périphérie du site, mais aussi à la périphérie du visible. Elle photographie les arbres de la forêt de Birkenau à la base de l'arbre, adoptant alors un point de vue qui n'est pas celui du promeneur mais celui du chercheur. On ne voit qu'une partie du tronc et le sol à sa base, en gros plan. Or quoi de plus banal qu'un arbre dans une forêt? Quoi de plus identique à un arbre qu'un autre arbre ? C'est à une même difficulté à interpréter la trace, à la considérer comme telle, qu'est confronté le spectateur face au travail de Guillaume Herbault. Les objets ne sont plus ici dissimulés au regard, ou dépouillés de leur sens par le choix de l'angle de vue. Dans sa série au titre énigmatique « $5 / 7 »^{18}$ le photographe affirme s'intéresser à " notre rapport à l'événement dramatique passé dans l'étude de ses effets présents »" Il explore ainsi différents territoires, dont Oswiecim, la ville qui abritait le camp de Birkenau en Pologne, où il ne photographie qu'une seule fois les structures du camp et s'intéresse surtout à la vie de cette ville aujourd'hui. Dans sa série Urakami, il va à la rencontre des survivants de la bombe de Nagasaki. Avec Slavoutich, il parcourt cette ville reconstruite après la catastrophe nucléaire de Tchernobyl afin de reloger les habitants de Pipriat, qui se trouve elle aussi contaminée. La catastrophe de Tchernobyl inspire d'ailleurs un livre au photographe, Tchernobylsky. L'ouvrage mêle les vues des lieux et des portraits. Ces portraits sont ceux des "condamnés à mort ", ces personnes encore vivantes mais irradiées, en sursis. En l'absence de séquelles flagrantes, évidentes de l'explosion, le photographe explore des espaces à l'abandon. L'événement est là, en filigrane, dont les seules traces sont ces objets qui ne disent rien en eux-mêmes, qui ne montrent rien de ce passé dramatique. Un mystère des lieux ${ }^{20}$ dû au fait qu'ils ne présentent pas le caractère visible de ruines, bien qu'ils en soient.

Certains lieux résonnent d'un écho encore plus faible. Ainsi en témoignent les photographies réalisées par Christophe Draeger, lequel revient sur les lieux de ces faits d'actualité qui ont occupé la une des journaux. Dans sa série Voyage apocalyptique, il 
s'attache tout particulièrement aux actes terroristes, aux catastrophes naturelles ou aux crashes aériens. Autant d'événements qui semblent surgir de nulle part, qui frappent aveuglément. Le photographe se rend sur les lieux des désastres, comme Three Miles Island, Lockerbie ou Kobe, et les photographie plusieurs années après les événements. Ce ne sont plus alors que des endroits communs, sans traces de dévastation. La gare de Bologne, par exemple, pourtant théâtre d'attentats terroristes sanglants, est à nouveau un lieu tout à fait ordinaire, avec des voies, des trains et des quais couverts. De l'incompréhensible violence, rien n'est conservé. La mémoire est comme effacée, on est face à une sorte de forclusion du paysage, qui se refuse comme lieu de résonance de la mémoire. Un camouflage du temps que constate Stéphane Duroy en parcourant L'Europe $d u$ silence $e^{21}$ à la recherche de traces des deux guerres mondiales qui ont déchiré le continent. Sa pérégrination le mène de la Pologne à l'Est de la France, en passant par Berlin. Mais il semble que cette recherche des stigmates des conflits reste infructueuse. C'est ce que la première image de son livre, représentant un paysage boisé baigné d'une lumière bleutée, nous laisse supposer. Le lieu quelconque, ou du moins non reconnaissable, semble annoncer que l'on ne verra rien d'extraordinaire. Reliant les lieux et les époques, Stéphane Duroy découvre des monuments commémoratifs qui semblent incongrus, voire incompréhensibles. Littéralement, on ne sait pas quel événement cette statue de cet ange de la mort commémore, comme on ne peut déchiffrer cette stèle photographiée plein cadre. Les inscriptions sont devenues illisibles, recouvertes d'une mousse noire, avec au centre une photographie presque effacée. Les autres images de la série représentent des rails vides de tout train, une femme se maquillant devant une fenêtre aux rideaux tirés, un angle de rue enneigé, une route par temps de brouillard, un intérieur baigné de rouge... Les lieux du drame nous paraissent pour la plupart quelconques.

18 Arno Gisinger insiste d'ailleurs sur ce point, affirmant que dans la « recherche du vide, le banal est de plus en plus important pour [lui] $\aleph^{22}$. La violence de ces images se révèle seulement dans le texte qui les accompagne.

\section{La « mise sous tension » de l'image}

19 La puissance évocatrice des images « en creux » semble contenue dans l'établissement d'une relation entre représentation iconographique et représentation linguistique. Le texte, en effet, n'ancre pas seulement le sens de l'image, mais se présente comme un élément de sa mise sous tension. Face à un certain mutisme de l'image, laquelle ne donne rien à voir en soi, le langage se fait moteur de l'acte de remémoration. Le texte nous renvoie à ce qui n'est pas visible, à « ce qui a été ", mais qui n'est plus. C'est dans l'association du texte et de l'image que l'actualité du drame refait surface. Le texte plonge le spectateur dans le théâtre de sa mémoire, soit par l'établissement d'un écart entre les deux formes du discours, linguistique et iconographique, soit à travers une identification du visuel par l'écrit.

Certains photographes jouent sur la distance, en établissant une relation distendue entre le texte et l'image. Stéphane Duroy et Sophie Ristelhueber, par exemple, travaillent sur ce double langage des mots et de l'image. Ni commentaires, ni descriptions des images, les textes formulent un écart signifiant, ils ouvrent une possibilité de sens sans être prescriptifs. Stéphane Duroy ponctue L'Europe du Silence de trois extraits, placés en ouverture, en milieu, et en conclusion de l'ouvrage. 
Leurs visages, pointus, duveteux et morts ont cette épouvantable absence d'expression de cadavres d'enfants. On se sent la gorge serrée quand on les voit bondir, courir et tomber. On voudrait les battre, parce qu'ils sont si bêtes, - et aussi les prendre dans ses bras et les éloigner de là où ce n'est pas leur place.

Erich Maria Remarque, À L'Ouest rien de nouveau.

Leur vie est courte mais leur nombre infini. Ce sont eux, les Muselmänner, les damnés, le nerf du camp; eux, la masse anonyme, continuellement renouvelée et toujours identique, des nonhommes en qui l'étincelle divine s'est éteinte, et qui marchent et peinent en silence, trop vides pour souffrir vraiment. On hésite à les appeler des vivants : on hésite à appeler mort une mort qu'ils ne craignent pas parce qu'ils sont trop épuisés pour la comprendre.

Primo Levi, Si c'est un homme.

La plupart des hommes et des femmes évolués du dix-neuvième siècle aurait rangé parmi les plaisanteries sinistres la prédiction selon laquelle la torture et le massacre n'allaient pas tarder à proliférer une fois encore dans l'Europe 'civilisée'. Il n'est rien de naturel dans notre condition présente. Il n'y a rien de particulièrement convaincant ou de digne dans le fait que nous admettons que 'tout est possible'. En fait, semblable état d'esprit abaisse et déforme le seuil d'indignation. [...]. Amorphe, envahissante, notre familiarité avec l'horreur représente pour l'humanité une défaite absolue.

George Steiner, Dans le château de Barbe-Bleue.

21 Les extraits de À L'Ouest rien de nouveau, roman d'Erich Maria Remarque et de Dans le château de Barbe-Bleue, livre de George Steiner, sont imprimés en bas de pages restées vierges d'images. Ils prennent donc formellement la forme de légendes, sans être pourtant mis en relation avec une image en particulier. Le deuxième texte, en revanche, extrait du livre Si c'est un homme de Primo Levi, encadre littéralement une image en grande partie floutée, prise sur le site d'un camp de concentration datant de la Seconde Guerre Mondiale. Sans ancrer véritablement le sens des images, ces textes donnent à voir le contexte de compréhension, orientent le sens de la lecture. Ils encadrent le livre, comme celui de Primo Levi encadre l'image du camp. On remarque d'ailleurs que la chronologie des textes respecte parfaitement celle de l'histoire, À l'Ouest rien de nouveau étant un roman allemand écrit sur la guerre de 1914, celui de Primo Levi traitant de la déportation qui a eu lieu pendant la Seconde Guerre Mondiale, le livre de Steiner étant un essai plus généraliste sur la question de la violence guerrière, proposant une vision rétrospective de notre siècle, sorte de conclusion après l'exposé des faits.

De la même manière, Sophie Ristelhueber ne mêle pas ostensiblement texte et image, mais encadre son ouvrage avec deux citations. Fait s'ouvre et se termine sur deux doubles pages, deux textes de Clausewitz qui se présentent sous la forme de ready-mades littéraires. Tout comme ses images en forme de all over, les textes semblent se continuer de part et d'autre de la page. Ainsi publiés, ces textes ne peuvent être considérés comme des extraits. Ce sont des fragments, arbitrairement extraits du corps du texte, au milieu d'une phrase.

... ne trouve d'ailleurs aucune difficulté à séparer l'une de l'autre, les diverses activités, lorsqu'on considère les forces armées et équipées comme des moyens donnés, dont il suffit pour s'en servir efficacement de connaître les principaux effets. Au sens strict, l'art de la guerre est donc l'art de savoir se servir au combat de moyens déterminés, et nous ne saurions mieux le désigner qu'en le nommant conduite de la guerre. Il est vrai, d'autre part, que l'art de la guerre, au sens plus large, englobe toutes les activités que suscite la guerre, par conséquent toute la création des forces armées, c'est-à-dire le recrutement, l'armement, l'équipement et l'entraînement. Pour que la théorie recouvre la réalité, il importe 
essentiellement de séparer ces deux activités, car on conçoit sans peine, si tout art de la guerre devait commencer par l'organisation des forces armées et leur coordination selon ses règles, que cet art ne serait applicable qu'aux rares occasions où les forces armées existantes correspondraient exactement à ces règles. Mais si l'on veut disposer d'une théorie qui s'applique à la grande majorité des cas sans être jamais tout à fait inutilisable, celle-ci doit se fonder sur le plus grand nombre de moyens de combat ordinaires et sur leurs effets essentiels. La conduite de la guerre est donc l'ordonnance et la conduite du combat. Si le combat consistait en une seule action, toute division supplémentaire n'aurait aucun sens. Mais le combat consiste en un plus ou moins grand nombre d'actions distinctes qui forment un tout et que...

La contiguïté de ces tranches d'un texte consacré à l'art de la guerre avec ces images arrêtées, figées sur ses effets, impose au spectateur un mode d'appréhension dédoublé. Les mots, énoncés d'une théorie guerrière, font échos aux images, représentations des ruines de l'après. Mais quand tout semble ordonné et précis à la lettre, le paysage est ravagé en profondeur. Sophie Ristelhueber donne ainsi au spectateur à lire et à voir, se démarquant ainsi d'une économie visuelle médiatique caractérisée par le précepte du voir sans lire. La photographe ici « tente de faire ressurgir des images permanentes ${ }^{23}$, attribuant par là une dimension universaliste à ses images, tout en s'éloignant de la commémoration ou même de la dénonciation.

Ainsi, par la déliaison du texte et de l'image, Sophie Ristelhueber comme Stéphane Duroy proposent au spectateur un axe de réflexion. Le spectateur est libre de tirer ses propres conclusions. Les images « en creux » restent latentes, en suspens. Néanmoins, cette dimension d'énigme est relativisée par la présence d'indications plus précises quant aux circonstances des prises de vues: Sophie Ristelhueber laisse apparaittre la mention «Koweit, 1991 », quand Stéphane Duroy ajoute en conclusion de son livre L'Europe du Silence un texte retraçant les différentes étapes de son voyage, et rappelant sa motivation première de revenir sur les traces de deux conflits européens.

L'identification du visuel par l'écrit est le mode le plus utilisé par les auteurs. Christophe Draeger légende de manière laconique les photographies, tranquilles en apparence, de son Voyage Apocalytique: Three Miles Island, Lockerbie, Kobe, Gare de Bologne... La relation entre l'image et sa légende est plus évidente : elle désigne les lieux de la prise de vue. Les noms de ces lieux, théâtres des événements tragiques résonnent immédiatement dans la mémoire collective. Le " creux » de l'image devient flagrant, notre mémoire étant chargée d'images, images de violences, de désastres ou de guerres.

Cette mention se fait discrète chez Marie-Jeanne Musiol. La photographe ne nomme le lieu qu'en seconde partie d'un titre appartement anodin: Dans l'ombre de la forêt. Mais lorsque le terme "Auschwitz-Birkenau » est énoncé, même entre parenthèses, la tragédie revient au cœur de l'image et plus aucun détail ne lui échappe. La mention du nom entraîne une transformation radicale du sens de la représentation.

Dans Hiroshima monument, Hiromi Tsuchida surajoute à cette détermination géographique des données consignées dans un ensemble de cartes et de plans. Le plan de la ville qui accompagne chaque photographie, est complété par la légende, qui mentionne systématiquement la nature du vestige photographié, arbre ou bâtiment, et sa distance par rapport à l'épicentre. Marquant ainsi une distance constatée, mesurable, le photographe donne à voir une autre distance, symbolique celle-ci, entre le silence de la représentation et la sourde mémoire dont elle est porteuse. Guillaume Herbault «mesure » lui aussi les effets de la catastrophe, d'une manière plus sibylline. 
Sur chaque image de son livre Tchernobylssty ${ }^{24}$ apparait un chiffre, sans d'autre explication. Il s'agit en fait de la dose de radiation mesurée sur le lieu à l'époque de la prise de vue. Mais, cela, le spectateur n'a les moyens de le comprendre qu'à la fin, quand l'auteur rappelle qu'un niveau normal de radiation se situe entre 10 et 20 microrems. Chaque photographie doit donc être lue à l'aune du niveau enregistré au moment de la prise de vue, qui va jusqu'à 1250 microrems pour la zone interdite de Pipriat. Les images muettes dévoilent derrière ce chiffre éloquent le silence lourd et oppressant d'une catastrophe omniprésente bien qu'invisible. Guillaume Herbault arrive ainsi à faire sentir la présence de l'impalpable, les effets de la radiation. Le regard n'est pas confisqué, tout au plus dirigé. L'image reste ouverte, le spectateur ne doit pas s'indigner, ou s'attendrir. La réflexion n'est pas de l'ordre du pathos, mais de l'ordre de la pensée.

28 Retour sur l'événement, retour sur les lieux de l'événement: les photographes posent ici la question cruciale de la transmission de l'expérience. Une problématique de la mémoire qui se dédouble, puisqu'il s'agit à la fois du lieu de l'image et de l'image du lieu. Dans les deux cas, selon que l'on considère de manière autonome la représentation en tant qu'objet, soit que l'on se concentre sur le lieu sujet de la représentation, la question reste celle de leur qualification en tant que « lieux de mémoire ».

29 Les lieux de mémoire naissent, selon Pierre Nora ${ }^{25}$, du sentiment de la perte d'une mémoire collective. C'est la dissolution de notre rapport au passé et la mise à distance de l'histoire qui rendent nécessaire la désignation de lieux porteurs de cette mémoire. Tant matériels qu'immatériels, physiques que symboliques, ces espaces de commémoration ne constituent pas la mémoire collective mais sont les instruments de sa réactivation. Tout comme les images "en creux" seraient les instruments de remémoration d'une mémoire collective constituée par les « images monuments». Si d'un point de vue historique on peut analyser l'apparition des images « en creux » dans le champ de l'art contemporain comme une réaction à la prolifération des icônes de la presse, elles n'en sont donc pas l'antithèse, au contraire. L'image " en creux » n'est donc pas une fin en soi, elle est le passage, l'intermédiaire entre le présent et le passé, entre le spectateur et la mémoire collective.

30 Il semble que le fait même suscite un engagement intellectuel et sentimental du spectateur, les disqualifie du point de vue du processus de transmission de la mémoire. Car alors la mémoire n'est plus vécue comme une évidence, elle n'est plus naturelle mais « outrageusement arquée par l'aliénation $»^{26}$ selon Philippe Dagen.

31 Un appel à la mémoire collective qui conserve toutefois une part d'aléatoire, du fait de la nature de la mémoire collective elle-même. Car bien qu'elle soit partagée, elle n'est pas uniforme. Si chaque individu construit effectivement sa perception du passé à partir d'images communes, il les intègre à sa propre mémoire et la forme de la remémoration est à chaque fois différente. Ainsi, on peut se demander dans quelle mesure cette forme de remémoration quelque peu aléatoire ne conduit pas à une trahison des faits, une mystification inconsciente. D'autre part, dans quelle mesure ces œuvres «fonctionnent-elles» si la mémoire collective change ou si elle se perd? Il s'agit là d'une problématique commune à tout lieu de mémoire, qui n'existe que «si l'imagination l'investit d'une aura symbolique ${ }^{27}$. Sans écho dans la mémoire collective, ces images sonnent creux.

32 Enfin l'image « en creux » n'opère pas seulement un retour sur la représentation de l'évènement, mais sur les lieux-mêmes de l'événement. À rebours du principe de 
simultanéité qui régit l'enregistrement photographique de l'événement, elles proclament non plus «j'y étais », mais «j'y suis allé ». Les photographes se détachent des lieux de commémoration attendus, organisés, pour aller à la recherche de la présence de ce passé dans notre quotidien. En photographiant des espaces vides de toute marque du passé, ils poussent le spectateur à s'interroger. Le regard balaie l'image à la recherche d'un signe, d'une trace, d'un symbole. Et il ne trouve que le trivial, le banal, le quotidien. Les images « en creux » tentent ainsi d'initier, à travers le sujet de la représentation, un mouvement de retour vers les milieux de mémoire. Le passé n'existe plus seulement dans le monument, lieux ou image, physique ou symbolique. Il n'est plus visible, mais invisible et ressenti seulement à travers l'expérience, à travers le vécu. Ces œuvres questionnent ainsi notre capacité à faire exister cet événement dans notre présent en découvrant la puissance de l'anodin dans notre rapport au passé.

\section{NOTES}

1. Pierre Nora, «Le Retour de l'événement ", in Faire l'Histoire, Nouveaux Problèmes, Jacques Le Goff et Pierre Nora, Paris, Gallimard, 1986, p. 212.

2. Vincent Lavoie, L'Instant-monument. Du fait divers à l'humanitaire, Montréal, Dazibao, 2001.

3. Le terme de "mémoire collective » est ici entendu au sens, très large, de mémoire collective occidentale. Il convient de considérer dans ce texte la notion de mémoire collective non comme une notion complète, en référence aux textes de Maurice Halbwachs, La Mémoire collective, Paris, PUF, 1950, mais plutôt comme un outil conceptuel. La mémoire collective, ou partagée, désigne ainsi la mémoire d'un ensemble d'individus.

4. Paul Ardenne, "L'Image d'art contemporain: impossible définition et stratégies de recomposition ", L'Art même $\mathrm{n}^{\circ}$ 27, 2005.

http://www2.cfwb.be/lartmeme/no027/pages/page4.htm.

5. Paul Ardenne, L'Image corps, Figures de l'humain dans l'art du XX $X^{e}$ siècle, Paris, Éditions du Regard, 2001, p. 471.

6. Jean-Paul Sartre, L'Imaginaire, Psychologie phénoménologique de l'imagination, Paris, Gallimard, 1964, p. 239.

7. Arno Gisinger, «La Photographie : de la mémoire communicative à la mémoire culturelle », in Mémoire des camps, Photographies des camps de concentration et d'extermination nazi (1933-1999), éd. Clément Cheroux, Paris, Marval, 2001, p. 182.

8. Michel Guerrin, « Mathieu Pernot, une vision en creux », Le Monde, 29 janvier 2005.

9. Dominique Baqué, «La Stratégie du retrait», in Photographie plasticienne, L'Extrême contemporain, Paris, Editions du Regard, 2004, p. 237-264.

10. Dominique Baqué, «L'ère du soupçon », Art Press n²73, novembre 2001, p. 42.

11. Jérôme Sans, «Une đuuvre de terrain », conférence prononcée le 22 novembre 1995 à partir de Fait de Sophie Ristelhueber, Caen, FRAC Basse-Normandie, 1995, p. 3.

12. On peut noter que Hiromi Tsuchida tend lui-même à lui donner une importance particulière. En effet, lorsqu'il édite Hiroshima Monument II en 1990, sorte de deuxième opus de l'œuvre, celui-ci ne comporte plus que des vues de la ville. Elles sont organisées en diptyques comparatifs, 
mettant en relation deux vues d'un même endroit: l'image réalisée en 1979, dont certaines publiées dans la première partie de Hiroshima Monument, et une vue prise en 1990.

13. Étienne Hatt, « Regardez avec moi... » , un entretien avec Arno Gisinger, Vite vu, Le Blog de la société française de photographie, mercredi 8 mars 2006. www.sfp.asso.fr/vitevu/index.php/ Entretiens.

14. Michael Fried, Absorption and Theatricality: Painting and Beholder in the Age of Diderot, 1980, tr. fr. La Place du spectateur, Esthétique et origines de la peinture moderne, Paris, Gallimard, 1990.

15. Étienne Hatt, « Regardez avec moi... » , un entretien avec Arno Gisinger, op .cit.

16. Michel Guerrin, «Les Obsessions de Sophie Ristelhueber », Le Monde, 27-28 septembre 1992.

17. Jérôme Sans, «Une Oeuvre de terrain », op. cit., p. 7.

18. Le titre de « $5 / 7$ », donné à ces séries lors de l'exposition «Croiser des Mondes » au Jeu de Paume à Paris en 2006, est en fait un titre provisoire. Il désigne une œuvre en sept parties, dont cinq seulement étaient réalisées à l'époque.

19. Guillaume Herbault, entretien avec Michel Poivert, Croiser des mondes, Document 2, Paris, éditions du Jeu de Paume, 2005, p. 46.

20. Ibidem, p. 46.

21. Stéphane Duroy, L'Europe du silence, Paris, Filigranes, 2000.

22. Etienne Hatt, « Regardez avec moi... » , un entretien avec Arno Gisinger, op. cit.

23. Jérôme Sans, « Une Oeuvre de terrain », op. cit., p. 9.

24. Guillaume Herbault, Tchernobylsty, Toulouse, Le Petit Camarguais/Privat, 2003.

25. Pierre Nora, "Entre Mémoire et Histoire, La problématique des lieux " in Les Lieux de la mémoire, La République, La Nation, Les Frances, éd. Pierre Nora, Paris, Gallimard, 1997.

26. Philippe Dagen, De Mémoires, Tourcoing, Le Fresnoy, Studio national des arts contemporains, Paris, Hazan, 2003, p. 9.

27. Pierre Nora, « Entre Mémoire et Histoire, La problématique des lieux » , op. cit., p. 37.

\section{RÉSUMÉS}

L'événement ne semble pas pouvoir exister s'il n'en existe pas d'images. Face à ce débordement, à ce «trop plein» visuel, on observe l'apparition d'une posture nouvelle dans le champ de la photographie contemporaine. Comme "en creux» du phénomène médiatique, ces images travaillent à l'évocation des grands évènements contemporains tout en étant vides de leur représentation explicite. Elles proposent un retour sur l'événement, un retour sur les lieux, pour tenter d'ouvrir un espace de réflexion sur, par, et dans l'image. Le «creux » n'est pas une représentation de l'absence ou de la disparition, mais du manque, celui de traces visibles de l'événement. Ces œuvres posent la question de la transmission de l'expérience tout en découvrant la puissance de l'anodin dans notre rapport au passé.

It seems an event cannot exist if images of them do not exist. Faced with this excess, this visual "overflowing", a new posture can be seen in the field of contemporary photography. As "negatives" of the media phenomenon these images evoke major contemporary events by not explicitly representing them. They propose a return to the event, a return to the scene, to try to open a space for reflection on, by, and in the image. The "negation" is not a representation of the absence or disappearance, but of the lack - the lack of visible traces of the event. These works 
raise the issue of the transmission of experience while uncovering the power of the commonplace in our relationship to the past.

\section{INDEX}

Thèmes : photographie

Index chronologique : XXe siècle

Index géographique : Europe, Japon

Mots-clés : actualités, Draeger (Christophe), Duroy (Stéphane), Ristelhueber (Sophie), Tsuchida (Hiromu)

Keywords : Draeger (Christophe), Duroy (Stéphane), news, Ristelhueber (Sophie), Tsuchida (Hiromu)

\section{AUTEUR}

\section{RAPHAËLE BERTHO}

EPHE - TU Dresden

Photographe et allocataire de recherche du collège doctoral européen "Ordres, institutionnels, écrits et symboles", en co-tutelle entre l'EPHE et la Technische Universität de Dresde, sa recherche porte sur la dimension sociologique et politique de la photographie contemporaine. Après avoir travaillé sur la photographie documentairee et sur les rapports entre la photographie et la mémoire collective, elle mène une recherche de doctorat sur les missions photographiques en Europe dans les années 1980 et 1990. Elle est chargée de cours au département de Médiation culturelle de l'université Paris 3. Elle a participé en tant que photographe à la Mission photographique du Conseil Général de Seine-Saint-Denis, puis coordonné pendant deux ans une mission photographique en collaboration entre l'Université Paris 8 et la Ville de Saint-Denis. 